\title{
COMPUTATIONAL SIMULATION OF BONE TISSUE REMODELING PROCESS A VIRTUAL TOOL FOR CLINICAL GUIDANCE
}

\author{
J. Belinha ${ }^{1,2}$, M.M.A. Peyroteo ${ }^{2,4}$, A.F. Oliveira ${ }^{3}$ and R.M. Natal Jorge 2,4 \\ ${ }^{1}$ Mechanical Engineering Department, School of Engineering, Polytechnic of Porto (ISEP), Porto, Portugal, job@isep.ipp.pt \\ ${ }^{2}$ Institute of Science and Innovation in Mechanical and Industrial Engineering (INEGI), Porto, Portugal, mmgomes@inegi.up.pt \\ ${ }^{3}$ Head of Orthopedics, Hospital Santo António - Hospital Center of Porto, Porto, Portugal, afonsecaoliveira1@gmail.com \\ ${ }^{4}$ Mechanical Engineering Department, Faculty of Engineering of the University of Porto (FEUP), Porto, Portugal, rnatal@fe.up.pt
}

Introduction: Bone is a living tissue, capable to structurally react to external stimuli, such as mechanical loading. In this work, bone tissue adaptation to mechanical loading is predicted by means of a friendly computational tool using an advanced topologic optimization mathematical model for bone remodeling.

Objectives: The aim of this work was to deliver a mature computational tool capable to predict the bone tissue remodeling in several scenarios, such as healthy or cancerous bone structures requiring implants.

Methods: In order to obtain the computational discretization of the bone structure, discrete numerical methods are used, such as the Finite Element Method and meshless methods. Then, the remodeling algorithm seeks the minimization of the strain energy density field and considers a phenomenological law capable to predict the bone tissue's mechanical properties based on the bone apparent density.

Finite Element and Meshless Method Analysis Software
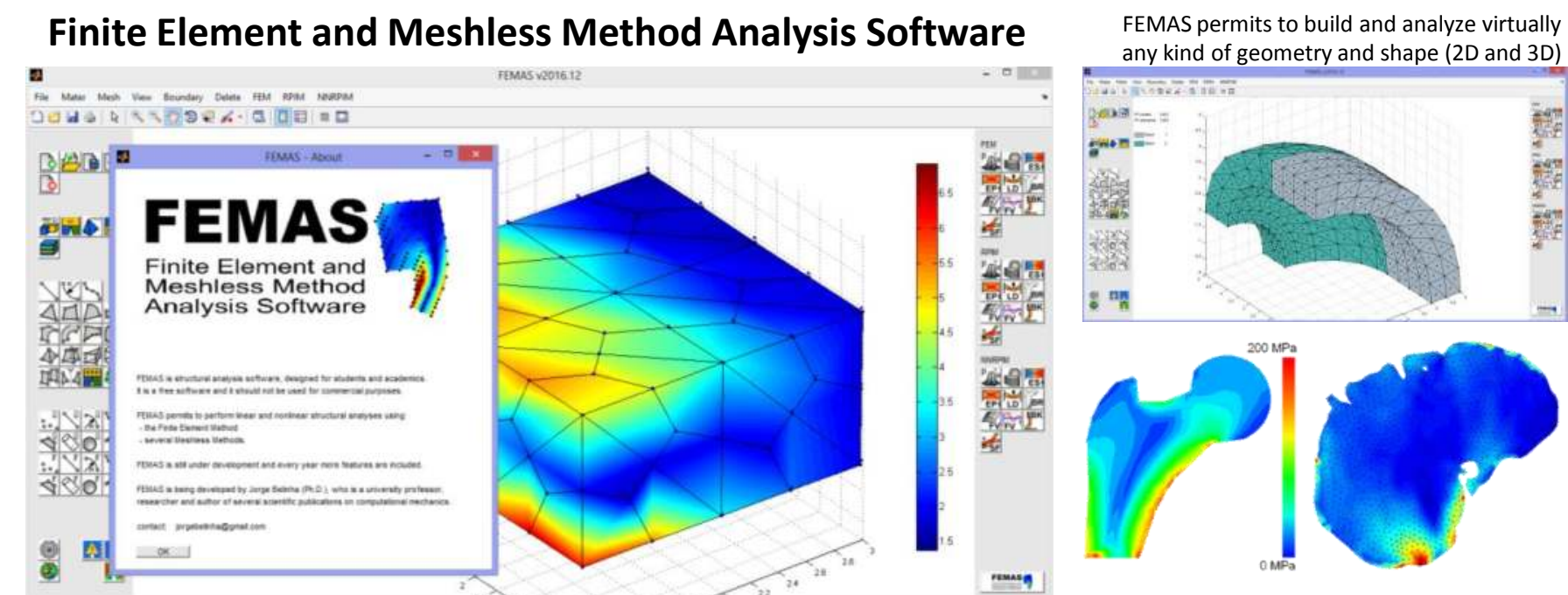

Results: In this work, several clinical cases are studied, such as the remodeling simulation of a femur submitted to a hip replacement surgery, whose head was removed due to a cancer. The virtual results show that, using the proposed computational tool, it is possible to predict the trabecular distribution of bone structures, with or without femoral stems.
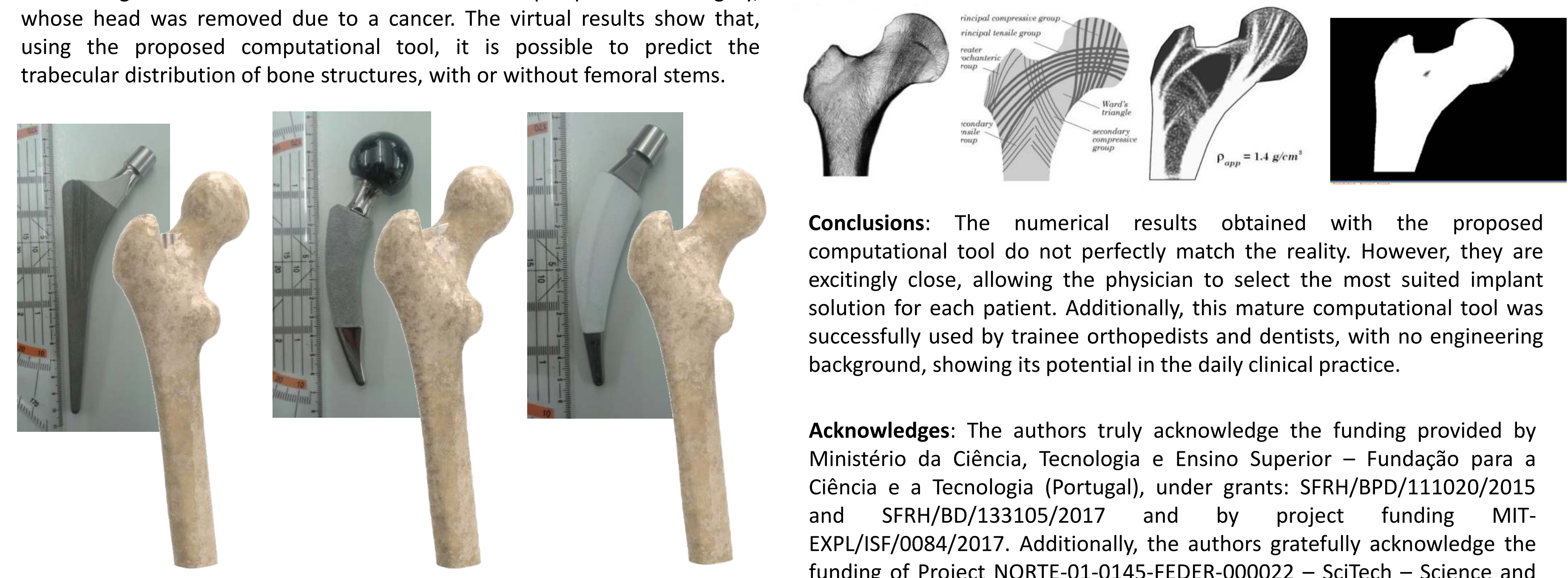

Conclusions: The numerical results obtained with the proposed computational tool do not perfectly match the reality. However, they are excitingly close, allowing the physician to select the most suited implant solution for each patient. Additionally, this mature computational tool was successfully used by trainee orthopedists and dentists, with no engineering background, showing its potential in the daily clinical practice.

Acknowledges: The authors truly acknowledge the funding provided by Ministério da Ciência, Tecnologia e Ensino Superior - Fundação para a Ciência e a Tecnologia (Portugal), under grants: SFRH/BPD/111020/2015 and SFRH/BD/133105/2017 and by project funding MITEXPL/ISF/0084/2017. Additionally, the authors gratefully acknowledge the funding of Project NORTE-01-0145-FEDER-000022 - SciTech - Science and Technology for Competitive and Sustainable Industries, co-financed by Programa Operacional Regional do Norte (NORTE2020), through Fundo Europeu de Desenvolvimento Regional (FEDER).

\section{References:}

[1] Belinha, J. (2014). Meshless Methods in Biomechanics - Bone Tissue Remodelling Analysis. (J.M. R. S. Tavares \& R. M. Natal Jorge, Eds.) (Vol. 16 ed.,p. 320). Lecture Notes in Computational Vision and Biomechanics, Springer Netherlands

[2] J.Belinha, L.M.J.S. Dinis and R.M. Natal Jorge (2016) "The analysis of the bone remodelling around femoral stems: a meshless approach". Mathematics and Computers in Simulation. Vol.121. pg.64-94. DOI: 10.1016/j.matcom.2015.09.002 